This is a postprint version of the following published document:

Deiss, Thomas, et al. Packet forwarding for heterogeneous technologies for integrated fronthaul/backhaul. EuCNC 2016, "The dawn of 5G", Papers (of the) European Conference on Networks and Communications, 27-30 June 2016, Athens, Greece. IEEE, 2016 (5 pp.)

DOI: https://doi.org/10.1109/EuCNC.2016.7561019

C 2016 by IEEE. Personal use of this material is permitted. Permission from IEEE must be obtained for all other uses, in any current or future media, including reprinting/republishing this material for advertising or promotional purposes, creating new collective works, for resale or redistribution to servers or lists, or reuse of any copyrighted component of this work in other works. 


\section{Packet Forwarding for Heterogeneous Technologies for Integrated Fronthaul/Backhaul}

Thomas Dei $\beta^{1, *}$, Luca Cominardi ${ }^{2,3}$, Andres Garcia-Saavedra ${ }^{4}$, Paola Iovanna ${ }^{5}$, Giada Landi ${ }^{6}, \mathrm{Xi} \mathrm{Li}^{4}$, Josep Mangues-Bafalluy ${ }^{7}$, Jose Núñez-Martínez ${ }^{7}$, Antonio de la Oliva ${ }^{3}$

${ }^{1}$ Nokia, ${ }^{2}$ InterDigital Europe, ${ }^{3}$ University Carlos III of Madrid, ${ }^{4}$ NEC Europe Ltd, ${ }^{5}$ Ericsson Telecomunicazioni, ${ }^{6}$ Nextworks, ${ }^{7}$ Centre Tecnològic de Telecomunicacions de Catalunya

\begin{abstract}
To meet the future mobile user demand at a reduced cost, operators are looking at solutions such as C-RAN and different functional splits to decrease the cost of deploying and maintaining cell sites. The use of these technologies forces operators to manage two physically separated networks, one for backhaul and one for fronthaul. To solve this issue, transport networks for 5G will carry both fronthaul and backhaul traffic operating over heterogeneous data plane technologies. Such an integrated fronthaul/backhaul (denoted as 5G-Crosshaul) transport network will be software-controlled to adapt to the fluctuating capacity demand of the new generation air interfaces. Based on a proposed data- and control-plane architecture for 5GCrosshaul, we propose a frame format common to both fronthaul and backhaul traffic as well as a corresponding abstraction of the forwarding behavior of the network elements. The common frame format and the forwarding abstraction define the information to be exchanged at the southbound interface (SBI) of the 5G-Crosshaul Control Infrastructure (XCI). This paper derives requirements for the SBI from $5 \mathrm{G}$ use cases.
\end{abstract}

Keywords-5G architecture, fronthaul, backhaul, network slicing, multi-tenancy, forwarding abstraction, SDN, NFV

\section{INTRODUCTION}

The transport networks for $5 \mathrm{G}$ will have to connect base stations or access points having different functional splits with the sites hosting baseband processing or general computing resources, see e.g. [1]. Therefore, both fronthaul and backhaul traffic will have to be carried across the same transport infrastructure, even along with the traffic of fixed access networks. On the other hand, $5 \mathrm{G}$ and beyond $5 \mathrm{G}$ Radio Access Networks (RAN) are expected to be technologically heterogeneous. They are also expected to be based on denser deployments to increase the spectrum reutilization and their capillarity. Therefore, this integrated fronthaul/backhaul transport network will be deployed over heterogeneous media and transport technologies, e.g. microwave, mmWave, dark fiber, leased lines, etc. With these requirements in mind we propose an adaptive, flexible and software-defined architecture for future $5 \mathrm{G}$ transport networks integrating multi-technology fronthaul and backhaul segments. This 5G-Crosshaul architecture aims to enable a flexible and software-defined reconfiguration of all networking elements through unified data and control planes interconnecting distributed $5 \mathrm{G}$ radio access

The authors of this paper have been sponsored in part by the project H2020 ICT-2014-2 "5G-Crosshaul": The 5G Integrated fronthaul/backhaul” (671598) *Corresponding author email: thomas.deiss@nokia.com and core network functions, hosted on in-network cloud infrastructure.

This paper presents the definition of a unified data-plane for the integrated fronthaul/backhaul transport network for $5 \mathrm{G}$. The general data plane architecture is described in Section II. It consists of interconnected switching entities called 5GCrosshaul Forwarding Elements (XFEs), featuring both circuitand packet-switching capabilities.

More specifically, if we assume optical input and output ports of the XFE, switching among optical media could both be done at the optical level, e.g. wavelength switching in a Dense Wavelength Division Multiplexing (DWDM) domain or circuit switching as in Optical Transport Network (OTN), as well as at the packet level in a similar way as Ethernet switches with fiber interfaces. In this way, strict latency/jitter requirements may be fulfilled through optical/circuit switching. Given the expected dynamicity and heterogeneity of $5 \mathrm{G}$ traffic, packet-based forwarding could be used to exploit the resource utilization efficiency and cost-effectiveness of statistical multiplexing.

In Section III, we present the 5G-Crosshaul Common Frame (XCF) format to be used across all segments (fronthaul and backhaul) of the network. This is a new frame format based on Ethernet defined to fulfil the heterogeneous requirements of fronthaul and backhaul. The XCF is one of the key contributions of 5G-Crosshaul towards an integrated 5G transport network. The impact of the proposed XCF on the abstract behavior of the forwarding elements is also described.

The 5G-Crosshaul Control Infrastructure (XCI) is summarized in Section IV. The XCI controls the transport network infrastructure (including network, compute, and storage elements) via its southbound interface (SBI).

As far as forwarding is concerned, both the XCF and the abstract forwarding behavior determine the information that has to be exchanged at the SBI of the XCI to manage and control the forwarding elements. Section $\mathrm{V}$ identifies candidates to define the forwarding-related functionality and the requirements for the SBI. Furthermore, and given the expected heterogeneity in transport technologies, the SBI will also feature technology specific information exchange. Finally, Section VI concludes the paper.

\section{DATA PlANE ARChITECTURE}

The 5G-Crosshaul data plane architecture comprehends an inter-connected packet-circuit switched transport network. The packet switched network is the primary path for the transport of most delay-tolerant fronthaul and backhaul traffic, whereas the circuit switched network (mainly optical) complements the packet switched path for those particular traffic profiles that 
are not suited for packet-based transport (e.g. legacy CPRI (Common Public Radio Interface) or traffic with extremely low delay tolerance). It can also be used for offloading the packet processing component of XFEs. This two-path switching architecture combines bandwidth efficiency, through statistical multiplexing in the packet switch, with deterministic latency guaranteed by the circuit switch. The 5G-Crosshaul network is composed of XFEs, which include packet switching (XPFE) and circuit switching elements (XCSE). This paper focuses on the packet switched network.

The 5G-Crosshaul switching elements use a common frame format $(\mathrm{XCF})$, which is presented in Section III. Adaptation functions (AF) map non-XCF-compliant fronthaul or backhaul traffic into the XCF format used within the packet switching network. An AF is e.g. needed for traffic originating at a remote radio head (RRH) and passing via the packet switching path to a base-band unit (BBU).

XPFEs are switching units that support single or multiple link technologies (e.g. mmWave, Ethernet, fiber, microwave, copper). As shown in Figure 2, a key part of the envisioned solution is a common switching layer in the XPFEs to enable a unified and harmonized traffic management. This common switching layer supports the $\mathrm{XCF}$ format across the various traffic types (of fronthaul and backhaul) and the various link technologies in the forwarding network..

\section{5G CROSSHAUL COMMON FRAME (XCF)}

The packet-switching part of the 5G-Crosshaul data plane needs to support heterogeneous links. The 5G-Crosshaul Common Frame (XCF) shall carry both fronthaul and backhaul data over such heterogeneous links. It is based on Ethernet frames, therefore relevant standards for packetized fronthaul are summarized before the XCF is presented.

\section{A. Recent Ethernet Extensions}

Several IEEE working groups have been extending Ethernet to handle time sensitive and fronthaul traffic. Some of the working groups have just started and the proposals are still subject to change.

Time Sensitive Networking (TSN) [2] is a collection of features in IEEE 802.1, which targets providing extremely low packet loss rates, finite, low and stable end-to-end latencies and synchronization among bridges and end stations:

Time synchronization to an accuracy of better than lus is achieved by IEEE 802.1AS: In such a system, all bridges and end stations have to be time aware. Besides Ethernet (802.3), 802.1AS can also be used e.g. for WiFi or Ethernet Passive Optical Networks.

QoS with low latency and high probability of packet delivery is defined in IEEE $802.1 \mathrm{CB}$. It provides redundancy by duplicating packets and sending them on different paths to a destination. IEEE 802.1Qcc improves the stream reservation protocol, by which end stations can reserve bandwidth for

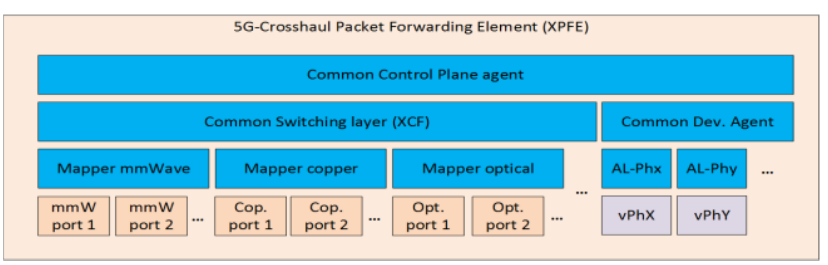

Figure 2: XPFE functional architecture traffic stream. IEEE $802.1 \mathrm{Qbv}$ extends the credit-based shaper by operations to open or close the gate for a traffic class. High priority traffic can be send as soon as its gate opens, i.e. it can be send with less jitter.

IEEE 802.1Qci provides protection against systems that could disrupt QoS or time synchronization by filter mechanisms based on packet rate and packet size at the boundary of a domain. This prevents misbehaving nodes to flood a network with too many or too large packets which in turn would break guarantees on latency and jitter.

IEEE $802.1 \mathrm{Qbu}$ allows express frames to preempt other frames. This reduces jitter for the express traffic.

IEEE 802.1CM [3] defines a profile to exploit these features to satisfy the latency requirements for fronthaul traffic, especially those of CPRI data.

IEEE 1904.3 [4] defines how CPRI data is mapped to Ethernet frames. Ethernet frames with CPRI data are indicated by a dedicated EtherType. The header of CPRI over Ethernet is part of the Ethernet payload. Two different mappings for CPRI data are defined: The structure agnostic mapping does not interpret the CPRI data in any way, it just puts the CPRI data to the Ethernet frame. The structure aware mapping differentiates between actual antenna data and e.g. control data and sends them in different Ethernet frames, which potentially may have different priority markings.

It is still under investigation which of the Ethernet features are used in the XPFEs. Frame preemption (802.1Qbu) is considered beneficial, where possible for the specific data link, also frame filtering (802.1Qci) to guard the network against malfunctioning nodes is considered beneficial.

\section{B. Common frame for heterogeneous technologies}

Circuit and packet switching may be deployed in 5GCrosshaul and are both controlled by the XCI. Even for packet switching alone, heterogeneous technologies may be deployed, such as Ethernet (IEEE 802.3), mmWave radio, WiFi (IEEE 802.11 ), etc. The XCF is used to transport data across such heterogeneous links.

The $\mathrm{XCF}$ is required to support multiple functional splits of the radio protocol stacks, i.e. traffic streams with diverse characteristics have to be transported in such frames. The XCF has to support prioritization as well as to prevent that these diverse traffic streams influence each other. The traffic streams may belong to different operators, therefore the XCF has to support multi-tenancy, keeping the streams of different tenants separate and allowing different forwarding behavior per tenant. The XCF has to be efficient, it should have low protocol overhead, allow the use of multiple paths towards one destination, and allow for multiplexing gains. Finally, the XCF has to be compatible with legacy technology and it has to be able to carry synchronization information.

Considering all the above requirements, a frame based on Ethernet has been considered as the best option for the XCF, more specifically on Provider Backbone Bridged Networks $(\mathrm{PBBN})$ frames or MACinMAC [5]. Basing the XCF on Ethernet allows using all the forthcoming extensions as described before. PBBN frames provide multi-tenancy support: the infrastructure provider can deploy multiple virtual networks and a tenant can provide additional separation within its network. The priority code points allow encoding different priorities, relevant to separate the types of traffic classes from 
each other. Eventually, a flow id can be used to direct different flows with same destination to different paths, while keeping each individual flow on the same path. The XCF can be used as well on WiFi based transport links, using the proposed standard amendment IEEE 802.11ak.

\section{CONTROL PlANE ARCHITECTURE}

The 5G-Crosshaul control plane architecture is centralized in the 5G-Crosshaul Control Infrastructure (XCI). The XCI provides control and management functions to operate the different types of resources composing the 5G-Crosshaul virtual and physical infrastructures, including both network nodes (incl. XFEs) and processing units (XPUs). Of particular interest in this paper is the control of the common switching layer present in the XFEs. In the following, we provide a highlevel overview of the XCI architecture.

The XCI hosts all the intelligence for the joint allocation and management of network and IT (compute and storage) resources. In addition to the management and orchestration building blocks common in the ETSI NFV architecture, at the lowest level, the network is mostly controlled by an SDN controller, whereas IT resources are managed using a Cloud controller. The SDN controller is responsible for the configuration of the network elements of the 5G-Crosshaul infrastructure (e.g., the XFEs), the cloud controller handles the 5G-Crosshaul IT components (computing and storage resources, i.e. the XPUs). Both the SDN and IT controllers provide a high level abstraction of the whole infrastructure to the orchestration layer inside the XCI, which is in charge of enabling the NFV building blocks to support the dynamic instantiation and allocation of virtual network functions throughout the $5 \mathrm{G}$ transport network. All the XCI functional entities can be implemented through proper extensions to opensource software: for example, OpenMANO or OpenBaton as baseline for NFV MANagement and Orchestration (MANO) components, OpenStack as Virtual Infrastructure Manager with OpenStack Nova as computing controller, and OpenDaylight or ONOS as SDN controller.

This paper focuses on a small part of the XCI, namely the interaction between the XCI SDN controller and the 5GCrosshaul network data plane at the Southbound Interface (SBI), which is detailed in section V. Further details on the XCI Northbound Interface (NBI) and the interaction between SDN controller and upper layer SDN applications for advanced network services like optimal end-to-end network provisioning for CDN (Content Delivery Networks) or TV broadcasting, network virtualization and multi-tenancy, energy optimization and mobility management are described in [6]. Further XCI interfaces at the East/Westbound side are also available towards the RAN and the Core Network domains, mainly for monitoring functions that enable inter-domain awareness.

A key feature of the XCI is its capability to handle multiple domains (technological or administrative domains). This is achieved by a hierarchical approach, by which lower control layers abstract their resources so that parent SDN controllers working with an end-to-end view do not need to deal with the specific details of each network region. Coordination between parent and child controllers is done through well-defined interfaces. Multi-technology domains may also be supported through multiple coexisting south-bound plugins within a single SDN controller. Each plugin implements a protocol specific southbound interface towards the data plane devices, while providing an abstract and unified view of the different network elements to the core services of the SDN controller.

The core services of the SDN controller, operating on top of the southbound plugins, offer northbound interfaces towards the SDN applications that enable an easy programmability of the entire network domains through REST APIs and information models, which are independent on the specific technologies at the data plane. In particular, these services provide inventory details about the capability of the network elements as advertised through the southbound interface, manage the automatic discovery and building of the physical network topology, collect and consolidate statistics data for monitoring and provide mechanisms for the configuration of the forwarding rules in each network entity managed by the controller. The combination of these services provides a further layer of abstraction at the whole network level, on top of the southbound plugins that, instead, operate at the level of single network entities.

\section{SOUTHBOUND INTERFACE}

This section details the main set of requirements, functionalities, and candidates for the 5G-Crosshaul southbound interface (SBI), enabling the interaction between the XCI and XFEs. As far as forwarding is concerned, the functionality of the SBI interfaces typically provide an abstraction of the switch's hardware providing node and forwarding models to enable direct expression of network behavior and requirements. In particular, SBI functionalities and information data models are on the one hand tightly coupled with the requirements of applications and use cases (see section V.A) and, on the other hand, constrained by the switch's backplane and transmission technologies. Usually, switch designers are free to implement the internal data models and procedures in any way convenient, as long as the switch preserves the correct match and instruction semantics defined by the SBI.

\section{A. Requirements from use cases and applications}

The requirements on the SBI are based on five key $5 \mathrm{G}$ use cases.

Dense urban society use case is focused on providing the connectivity required at any place and at any time by humans in dense urban environments composed of heterogeneous transport technologies in a mesh topology. It requires the SBI to support different transport technologies in a multi-domain and multi-vendor environment and to enable technology specific information exchange at the SBI.

Multi-tenancy use case aims to address the flexible sharing of Crosshaul resources across multiple tenants to reduce significantly CAPEX and OPEX by jointly sharing the infrastructure resources in a cost-efficient way. It requires the support of tenant awareness at the SBI to provide per tenant monitoring of network QoS and resources usage.

Vehicle mobility use case cares for the support of $5 \mathrm{G}$ communication in vehicles during motion, e.g. passengers using $5 \mathrm{G}$ services such as real-time video on a very high speed train (about $500 \mathrm{~km} / \mathrm{h}$ ) or messages among vehicles for traffic control, emergency and safety. The challenge of this use case 
on the SBI is to control high mobility to a crowd of passengers (e.g. more than 500 people) in a seamless manner.

Media distribution use case is related to the distribution of media contents over $5 \mathrm{G}$ networks, especially $\mathrm{CDN}$ and $\mathrm{TV}$ broadcasting. The SBI is required to provide a topology view for a CDN and broadcasting network.

Mobile Edge Computing (MEC) use case is focused on the deployment of IT and cloud-computing capabilities within the RAN, especially at the edge of the network in close proximity to mobile subscribers. The SBI needs to support NFV related configurations of VMs for dynamic VNF placement.

All these use cases require the SBI to provide an abstraction of the underlying network topology by providing nodes (XFEs, XPUs, etc.) and forwarding behaviors, as well as performance-, resource-, and energy-related information to enable a systemwide optimization of QoS, energy usage, cost, etc. to create the $5 \mathrm{G}$ transport services.

The SBI has to be sufficiently expressive to support a set of applications at the NBI of the XCI. These applications are explained further in [6]. The Resource Management Application (RMA) provides logically centralized and automated management of physical and virtual 5G-Crosshaul resources (computing, storage, and networking) to promptly provision transport services according to their SLA. The Multitenancy application (MTA) enables a generalized, flexible sharing of $5 \mathrm{G}$-Crosshaul infrastructure by multiple network operators leveraging virtualization techniques. The Mobility Management Application (MMA) deals with mobility support and mobile traffic offload for vehicle mobility and CDN use cases. The Energy Management and Monitoring Application (EMMA) deals with monitoring energy parameters of RAN, fronthaul, and backhaul elements, estimating and optimizing the energy consumption of the virtual 5G-Crosshaul network, while maintaining QoS for each tenant. The $C D N$ Management Application (CDNMA) and Broadcast Application (BA) applications are defined for media distribution services. The Virtual Infrastructure Manager \& Planner Application (VIMaP) enables other applications (such as MTA) to request the constrained allocation of physical and virtual 5G-Crosshaul resources represented as an abstracted construct, and proceeds to instantiate, deploy and provision them over the 5GCrosshaul infrastructure.

These applications require a specific set of information to operate. The XCI extracts such information from the data plane via the SBI. Table 1 summarizes the list of SBI requirements required by the $5 \mathrm{G}-\mathrm{Crosshaul}$ applications.

\section{B. SBI Candidates}

Based on the previous requirements, a set of state-of-the-art SBIs protocol candidates are considered as baseline in $5 \mathrm{G}-$ Crosshaul for controlling the forwarding behavior on the switches. Each of the candidates provides already an abstraction of the behavior of forwarding elements and links.

1) OpenFlow Protocol

OpenFlow [7] adopts a match-action model on the switches based on the concepts of flow table, flow entry, and pipeline. An OpenFlow switch includes one or more flow tables, interconnected in a pipeline, and flow entries, stored in the flow tables. Each flow entry consists of a matching rule, which
Table 1 - SBI requirements from applications

\begin{tabular}{|l|l|}
\hline SBI Requirements & Applications \\
\hline $\begin{array}{l}\text { Abstraction of underlying topology (virtual or } \\
\text { physical) represented by a list of nodes, links, } \\
\text { ports, capability in terms of networking and IT } \\
\text { resources, configuration parameters, etc. }\end{array}$ & $\begin{array}{l}\text { Required by all } \\
\text { applications }\end{array}$ \\
\hline $\begin{array}{l}\text { Per tenant monitoring of network QoS and } \\
\text { resources usage and tenant/operator awareness. }\end{array}$ & MTA \\
\hline $\begin{array}{l}\text { RAN configuration, e.g. RAN functional split, } \\
\text { BBU/XPU associated to each RRH, a set of RAN } \\
\text { functions offloaded into BBUs, XPUs, etc. }\end{array}$ & RMA \\
\hline $\begin{array}{l}\text { Dynamic configuration of forwarding behavior } \\
\text { of single nodes, for different flow granularities. }\end{array}$ & $\begin{array}{l}\text { Required by all } \\
\text { applications }\end{array}$ \\
\hline $\begin{array}{l}\text { Energy-related information of physical devices. } \\
\text { This could be dynamic, e.g. traffic load and } \\
\text { harvested energy, or static, e.g. available power } \\
\text { states, types of harvesting and energy storage. }\end{array}$ & EMMA \\
\hline $\begin{array}{l}\text { Monitoring report for the measured link QoS and } \\
\text { node performance in terms of resource usage on } \\
\text { computation and memory. }\end{array}$ & $\begin{array}{l}\text { Required by all } \\
\text { applications }\end{array}$ \\
\hline $\begin{array}{l}\text { Configuration of technology related parameters } \\
\text { such as transmission power, modulation, } \\
\text { channels, bandwidth, etc. }\end{array}$ & RMA, EMMA \\
\hline $\begin{array}{l}\text { Link performance events, such as sudden change } \\
\text { on links' throughput load, delay and loss, etc. }\end{array}$ & $\begin{array}{l}\text { MTA, RMA, } \\
\text { EMMA, VIMaP }\end{array}$ \\
\hline $\begin{array}{l}\text { Node performance events, such as sudden change } \\
\text { on energy consumption, computational load and } \\
\text { memory usage. }\end{array}$ & $\begin{array}{l}\text { MTA, RMA, } \\
\text { EMMA, VIMaP }\end{array}$ \\
\hline $\begin{array}{l}\text { Virtual/physical link state events: } \\
\text { Link failure and type } \\
\text { - Link up/down }\end{array}$ & $\begin{array}{l}\text { Required by all } \\
\text { applications }\end{array}$ \\
\hline $\begin{array}{l}\text { Virtual/physical node state events: failure and type } \\
\text { NMA, RMA, }\end{array}$ \\
\hline
\end{tabular}

defines the set of header fields or metadata used to match ingress packets, and one or more actions, applied to the matching packets. Actions can e.g. modify the packet header, send the packet to an output port or another flow table, or discard the packet.

\section{2) IETF ForCES}

The ForCES Forwarding Element (FE) [8] is a logical entity that implements the ForCES Protocol [9] and uses the underlying hardware to provide per-packet processing and handling as directed by a Control Element [10]. Logical Function Blocks (LFB) are the basic building block that reside on the FEs and are a functional abstraction of the FEs' processing capabilities. Packets coming into a FE generally flow through one or more LFBs before leaving at the egress ports. Several LFBs can be connected together to create a topological relationship. Such a topology is a directed graph representation of the logical data paths within an FE, the nodes representing the LFB instances, and the directed link depicting the packet flow direction from one LFB to the next.

3) $P 4$

$\mathrm{P} 4$ [11] is a high-level language for programming protocolindependent packet processors based on the concepts of reconfigurability, protocol independence, and target independence. Reconfigurability allows the controller to redefine the packet parsing and processing in the field. Protocol independence allows the controller to specify a packet parser for extracting header fields and a collection of typed match- 
action tables that process these headers. Target independence means that packet-processing functionality is independent of the specifics of the underlying hardware. These specifics are considered when compiling a P4 description to the hardware.

\section{Forwarding Abstraction}

An SBI provides an abstraction of the switch's hardware towards the network controller to enable direct expression of network behavior and requirements. Multiple abstraction models can be adopted depending on the involved functionality, e.g., a forwarding model defines the packet processing semantic on the switch, while a node model defines the device and peripherals abstraction.

An OpenFlow network node is modelled through several network ports and a pipeline composed of a set of flow tables [7]. The forwarding behavior of an OpenFlow switch is abstracted by describing how the different flows entering the switch travel along this pipeline and the type of actions which can be applied to the packets in the different flow tables. The set of match operations and actions is fixed and no further description of them has to be exchanged at the SBI.

In contrast, reconfigurable match operations and actions as in $\mathrm{P} 4$ require that the description of these operations is exchanged at the SBI. Both approaches allow to abstract and program behavior of a network element in a very flexible way and to achieve deep granularity in the packet classification. The flexibility of P4 however increases the cost of pipeline processing. Depending on the desired granularity of the flow description, it may require the analysis of the entire packet header to classify the flows and select the proper flow entry in each table. In environments with strict delay requirements, like a 5G-Crosshaul infrastructure, this can be a limitation and we propose to restrict the portion of packet header to be considered during the forwarding decision such that the packet processing speed can be optimized. This shows that depending on the chosen forwarding abstraction, different type of information has to be exchanged at the SBI. Although P4 provides high flexibility regarding the packet headers, this is not needed in 5G-Crosshaul due the choice of a common frame format. Furthermore, given the popularity, maturity, and the extensions defined (or under definition), OpenFlow provides the required flexibility for the project whilst increasing its innovation potential.

\section{Technology Specific SBI information}

The 5G-Crosshaul data plane supports multiple transmission technologies. Most parameters can be considered as common for any technology, but there are also technology specific parameters. All these need to be supported and abstracted by the SBI to enable the common control in the XCI. Three sets of parameters for monitoring, configuration, and inventory for each transmission technology will be considered.

In case of mmWave, where IEEE 802.11ad is used as MAC protocol, each node may have multiple sectors that need to be configured separately by the XCI. For example, a nonexhaustive list of parameters includes channel central frequency and bandwidth, noise threshold, beamwidth, modulation coding scheme, transmission power, retransmission policy, aggregation, and scheduling that determines the split of the total available bandwidth among the devices connected to the same sector. To enable the common control, the SBI may extend the concept of virtual port where each device connected to the mmWave node is seen as a different input/output port. By adopting this abstraction, a mmWave node can be represented as an OpenFlow switch with extended port description, modification, and statistics.

In optical networks, a different parameter list and modeling should be considered [12]: fiber type and dispersion, dispersion tolerance, ROADM capabilities and constraints, supported slot width granularity, amplifier parameters, and wavelength configuration. Similarly to the mmWave case, different wavelengths can be represented as different virtual ports by the SBI making the node OpenFlow-compliant.

Similar parameters and abstractions can be defined for other transmission technologies, such as copper and microWave. In addition to those port-related parameters, a further set of node-related parameters specific to the $5 \mathrm{G}$ Crosshaul network requirements should be considered as well : node and link latency specified for upstream and downstream and E2E latency are relevant to compute paths with low latency. It is relevant to indicate for each technology how to derive such values and make them available to the XCI.

\section{CONCLUSION}

In this paper we proposed an inter-connected packet-circuit data plane architecture and corresponding control infrastructure. Specifically, for the packet switching part of the data plane we proposed the XCF to carry data across heterogeneous links in both fronthaul and backhaul as well as the impact of the forwarding abstraction on the SBI of the control infrastructure.

\section{REFERENCES}

[1] Small Cell Virtualization Functional Splits and Use Cases, Document 159.05.1.01, Small Cell Forum, June 2015

[2] IEEE Time Sensitive Networking Task Group, http://www.ieee802.org/1/pages/tsn.html.

[3] IEEE $802.1 \mathrm{CM}$ - Time-Sensitive Networking for Fronthaul, http://www.ieee802.org/1/pages $/ 802.1 \mathrm{~cm} . \mathrm{html}$

[4] IEEE 1904.3, Standard for Radio Over Ethernet Encapsulations and Mappings, http://www.ieee1904.org/3/tf3_home.shtml.

[5] IEEE Std 802.1Q $\mathrm{Q}^{\mathrm{TM}}-2014$, Bridges and Bridged Networks. IEEE

[6] Xi Li, et.al. 2016, Innovations Through 5G-Crosshaul Applications, EUCNC 2016

[7] Open Networking Foundation (ONF), "OpenFlow Switch Specification," $2015 . \quad$ March Anline]. Available: https://www.opennetworking.org/images/stories/downloads/sdnresources/onf-specifications/openflow/openflow-switch-v1.5.1.pdf.

[8] J. Halpern and J. H. Salim, "Forwarding and Control Element Separation (ForCES) Forwarding Element Model," March 2010. [Online]. Available: https://tools.ietf.org/html/rfc5812.

[9] A. Doria, et.al, "Forwarding and Control Element Separation (ForCES) Protocol Specification," March 2010. [Online]. Available: https://tools.ietf.org/html/rfc5810.

[10] L. Yang, R. Dantu, T. Anderson and R. Gopal, "Forwarding and Control Element Separation (ForCES) Framework," April 2004. [Online]. Available: https://tools.ietf.org/html/rfc3746.

[11] Pat Bosshart, et.al. 2014. P4: programming protocol-independent packet processors. SIGCOMM Comput. Commun. Rev. 44, 3 (July 2014), 8795. DOI=http://dx.doi.org/10.1145/2656877.2656890

[12] ITU-T Rec. G.680, "G.680 : Physical transfer functions of optical network elements", 2007 